\title{
Hepatocellular carcinoma: cost-effectiveness of screening. A systematic review
}

\author{
This article was published in the following Dove Press journal: \\ Risk Management and Healthcare Policy \\ 18 June 2012 \\ Number of times this article has been viewed
}

\author{
Matteo Ruggeri \\ Facoltà di Economia, Università \\ Cattolica del Sacro Cuore - sede di \\ Roma, Rome, Italy
}

\begin{abstract}
Hepatocellular carcinoma (HCC) is one of the most common tumors worldwide. $\mathrm{HCC}$ is a potential target for cancer surveillance (or screening) as it occurs in well-defined, at-risk populations. Curative therapy is possible only for small tumors and screening strategy has been recommended by the US, Italian, and other international liver societies and is practiced widely, but its benefits are not clearly established. The objective of this study was to review the available evidence with respect to the cost-effectiveness of key technologies in the prevention HCC. The literature search was conducted with the support of PubMed. Firstly we selected articles by reading the abstracts. Secondly, we read the articles and the revision was further restricted, with the following as inclusion criteria: (1) full economic evaluation of HCC screening programs; (2) comparison between HCC techniques; (3) outcome measures expressed in terms of quality adjusted life years (QALY); (4) full text availability. The initial review of the literature yielded 346 articles. Of those, 288 were excluded at the first stage. Of those excluded, 108 did not meet the target, 106 did not present the cost analysis, 33 did not analyze the treatment of the disease, and in 41 the abstract was not available. Of the 58 included in the first step, seven examined the cost-effectiveness of different HCC screening techniques, seven investigated the cost-effectiveness of HCC screening versus no screening, and one looked at the cost-effectiveness of timing for HCC surveillance and monitoring, while 43 were about HBV vaccination and screening. We included only the seven articles examining the cost-effectiveness of different HCC screening techniques. In general, incidence is the key parameter which determines the cost-effectiveness of HCC screening. Discrepancies in the results exist when determining the type of technology to be used. Ultrasound (US) alone or in association with alpha fetoprotein (AFP) technology is likely to be the most cost effective and the use of computed tomography (CT) gives controversial results.
\end{abstract}

Keywords: HCC screening, economic evaluations, systematic review

\section{Introduction}

Hepatocellular carcinoma (HCC) is one of the most common tumors worldwide. The epidemiology of HCC exhibits two main patterns, one in North America and Western Europe and another in non-Western countries: sub-Saharan Africa; central and southeast Asia; and the Amazon basin. Males are affected more than females and its spread is most common between the ages of 30 to $50 .{ }^{1}$ HCC causes 662,000 deaths worldwide per year ${ }^{2}$ and half of them are in China. Most cases of HCC are secondary to either a viral infection (hepatitis B or C) or cirrhosis (alcoholism being the most common cause of hepatic cirrhosis). ${ }^{1}$ HCC may present with jaundice, bloating from ascites, easy bruising from blood clotting abnormalities or as loss of appetite, unintentional
Correspondence: Matteo Ruggeri Facoltà di Economia, Università Cattolica del Sacro Cuore - sede di Roma I.go F.Vito I, 00168 Rome, Italy Tel +39630156096

Email mruggeri@rm.unicatt.it 
weight loss, abdominal pain (especially in the upper right part), nausea, emesis, or fatigue. ${ }^{1-3}$

HCC is a potential target for cancer surveillance (or screening) as it occurs in well-defined, at-risk populations and curative therapy is possible only for small tumors. Surveillance has been recommended by regional liver societies $^{4}$ and is practiced widely, but its benefits are not clearly established. Hepatic ultrasonography (US) with or without alpha fetoprotein (AFP) performed every 6 months is the preferred program. Surveillance of HCC has been shown to detect small tumors for curative treatment, which may be translated to improved patient survival. However, most studies ${ }^{5-7}$ are limited by lead-time bias, length bias for early diagnosis of small HCC, different tumor growth rates, and poor compliance with surveillance. Cost-effectiveness of surveillance programs depends on the rate of small HCC detected "accidentally" (routine imaging) in a comparator group, annual incidence of HCC with various etiologies, patient age, and the availability of liver transplantation. The incremental cost-effectiveness for 6-monthly AFP and US has been estimated at approximately $\$$ US26,000-\$US74,000/ quality adjusted life years (QALY). ${ }^{8}$ All cirrhotic patients are therefore recommended for HCC surveillance unless the disease is too advanced for any curative treatment. As chronic hepatitis B and C can develop into $\mathrm{HCC}$ without going through liver cirrhosis, high-risk non-cirrhotic chronic hepatitis B and C patients are also recommended for HCC surveillance. HCC surveillance could be effective in reducing disease-specific mortality with acceptable cost-effectiveness among selected patient groups, provided it is a well-organized program. The objective of this study was to review the available evidence with respect to the cost-effectiveness of key technologies in the prevention HCC. The study focused on the techniques of economic evaluations based on decision models, with reference to early diagnosis.

\section{Methods}

\section{Methods for research}

The literature search was conducted with the support of PubMed. The Medical Subject Heading (MeSH) term used in the search string was "hepatocellular carcinoma." Hepatocellular carcinoma is defined in the MeSH as a "primary malignant neoplasm of epithelial liver cells." It ranges from a well-differentiated tumor with epithelial cells indistinguishable from normal hepatocytes to a poorly differentiated neoplasm. The cells may be uniform or markedly pleomorphic, or form giant cells. Several classification schemes have been suggested. The search string used was : "hepatocellular carcinoma" and "screening" and "economic evaluation" or ("cost of illness", "costminimization analysis", "cost-effectiveness analysis", "cost utility analysis", "cost benefit analysis"). During the search we applied the following limits:

1. language (languages): Italiano (Italian), English (English);

2. period of time (dates): from $01 / 01 / 00$ to $31 / 03 / 11$;

3. species (species): humans (humans).

\section{Selection of the studies}

The studies were selected by following a two-stage procedure. In the first stage the studies were selected by reading the abstract. When the abstract did not provide the information necessary to allow selection or exclusion, we proceeded to read the entire article. We followed as exclusion criteria:

1. studies not consistent with the objective;

2. only information on the treatment of hepatitis B, C and HCC;

3. abstract not available;

4. studies not showing cost analysis.

In the second stage, we read the articles selected in the first stage and the revision was further restricted to papers satisfying the following inclusion criteria:

1. full economic evaluation of HCC screening programs;

2. comparison between $\mathrm{HCC}$ techniques (thus excluding cost-effectiveness of HCC screening versus HCC treatment and cost-effectiveness of the timing of surveillance and monitoring);

3. outcome measure expressed in terms of QALYs;

4. full text available.

Figure 1 reports the steps of the literature search.

\section{Collection and data analysis}

The study data were retrieved by reading the entire article. The information obtained was summarized using an Excel (Microsoft, Redmond, WA) worksheet. Papers were reported in a table with the following fields: article title; name of first author; publication year; journal; type of study. In a second step, data on economic evaluations of screening programs were summarized in a table which contained the following fields: name of first author and year of publication; type of economic evaluation performed in the study (ie, cost effectiveness analysis) nation in which the study was conducted and perspective (third party payer/hospital); sample number and time horizon considered; measures of cost/ effectiveness measures (ie, cost/QALY); and results. 


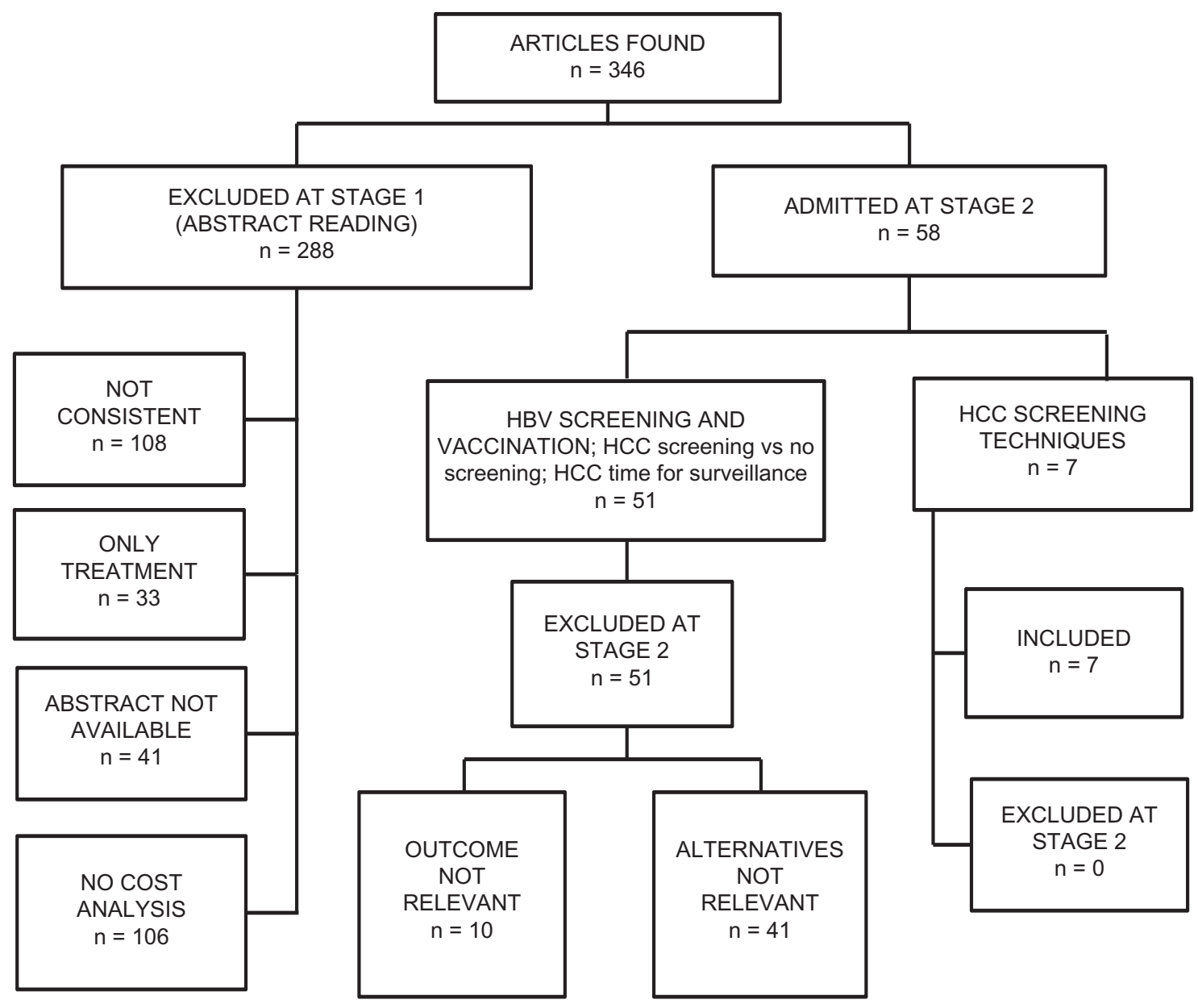

Figure I Results of the literature search.

\section{Results}

The initial review of the literature yielded 346 articles. Of those, 288 were excluded at the first stage. Of those excluded, 108 did not meet the target, 81 did not present the cost analysis, 31 did not analyze the treatment of the disease and in 41 the abstract was not available. Of the 58 included in the first step, seven examined the cost-effectiveness of different HCC screening techniques, seven investigated the cost-effectiveness of HCC screening versus no screening and one looked at the cost-effectiveness of timing for HCC surveillance and monitoring, while 43 were about $\mathrm{HBV}$ vaccination and screening. We included only the seven articles on the cost-effectiveness of different HCC screening techniques. The years of publication ranged from 2000 to 2011. The studies were conducted mainly in Europe and in the USA. The studies conducted to evaluate the cost-effectiveness of screening for HCC displayed frequent discrepancies in the results, probably due to differences in the study design, timing of execution of the tests, and cut-offs used. Most studies used decision models (Markov chain or decision tree) while trials were presented by a smaller number. The target population was represented by patients at risk of $\mathrm{HBV}$ and $\mathrm{HCV}$, with cirrhosis at an advanced stage, subject to the monitoring test or screening test in different settings (USA, UK, Italy, Australia, Taiwan, and other countries). All the studies took into account the direct costs related to screening programs. Concerning the measures of effectiveness, most studies expressed the outcomes in terms of QALY or LY. The complete results of the search are shown in Table 1.

In the decision tree model of Thompson et al, ${ }^{9}$ three strategies are compared: (1) US + AFP, (2) AFP triage and (3) US. The analysis suggests that, in patients with cirrhosis, surveillance strategies for HCC are effective and often are likely to be cost-effective. The most cost effective strategy is US + AFP (incremental cost-effectiveness ratio [ICER] $=£ 20.700 /$ QALY). The model estimated that, compared with no surveillance, this strategy may triple the number of people with HCC diagnosis. Two Markov models have been proposed to assess the cost-effectiveness of screening based on US, AFP, and CT (computed tomography). 


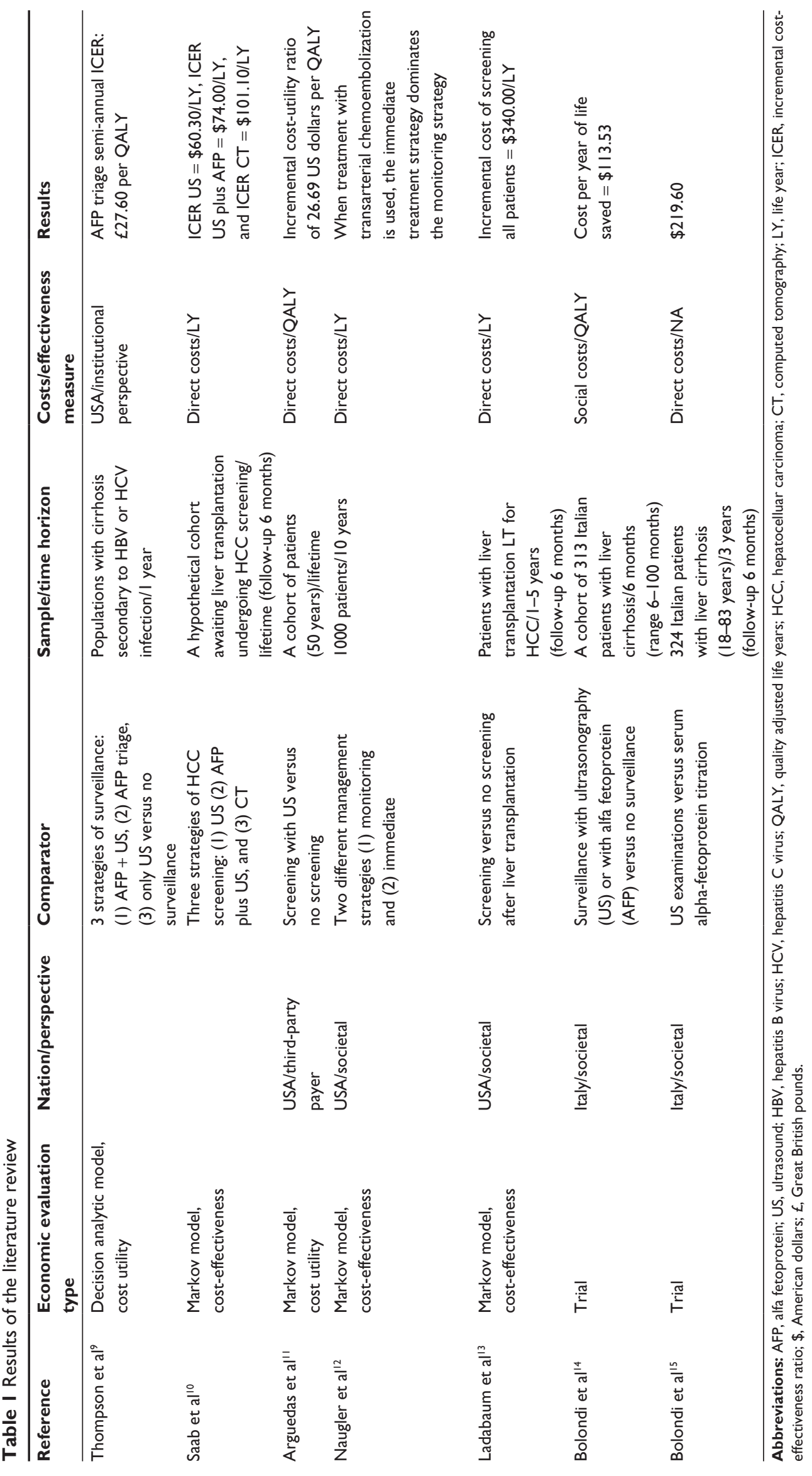


The Markov model proposed by Saab et a $1^{10}$ compares three different screening strategies: (1) US, (2) US + AFP, and (3) $\mathrm{CT}$. The screening is performed for all strategies at intervals of 6 months. The US screening strategy appears to be the most cost-effective. Arguedas et al ${ }^{11}$ have constructed a Markov model on a cohort of 50 patients with hepatitis C-related cirrhosis. The model shows an incremental cost-utility ratio of \$26,689 per QALY for screening with US, while three-phase CT AFP screening is associated with an incremental costutility ratio of $\$ 25,232$ per QALY versus no screening. The sensitivity analysis showed that results were more sensitive to the incidence of HCC. Screening for HCC with CT has proved to be cost-effective in patients eligible for transplantation with cirrhosis secondary to chronic hepatitis $\mathrm{C}$ virus (HCV) with respect to the other screening interventions. A study by Naugler et $\mathrm{al}^{12}$ compared survival rates and the cost-effectiveness of two screening strategies for small cell carcinoma: immediate treatment and monitoring. The first screening strategy provided transarterial chemoembolization (TACE) or radiofrequency ablation (RFA) and monitoring every 3 months with subsequent treatment. The other strategy involved monitoring every 3 months without treatment. With TACE, the monitoring strategy saved more lives than the strategy of immediate treatment (4.324 compared with 4.269 years of life on average), with a cost of $\$ 739,602$ per life year gained. Ladabaum et $\mathrm{al}^{13}$ have presented a Markov model to evaluate the cost-effectiveness of screening of HCC after liver transplantation. Screening is based on CT, AFP and chest radiograph (CR). The study results suggest that small gains in life expectancy can be achieved by screening after transplantation, possibly associated with relatively high incremental cost per life year gained.

A study by Bolondi et al ${ }^{14}$ involved 313 patients with cirrhosis who underwent a 6 month program of surveillance US and AFP. The surveillance program for patients required a large number of resources and offered little benefit in terms of patient survival. The total cost of the monitoring program was $\$ 753,226$ and the cost per treatable HCC was $\$ 17,934$ with a cost per life year saved of $\$ 112,993$. The annual cost of this program over a period of follow-up of 56 months was $\$ 175,314$. The results showed that this program required a large number of resources. Its implementation in clinical practice on a national basis is not readily accessible in many countries and depends on the prevalence of the disease in the population and the resources available within different settings. Finally, a study by Bolondi et $\mathrm{al}^{15}$ focused on evaluating the cost-effectiveness of a screening program for early diagnosis and treatment of $\mathrm{HCC}$ in patients with liver cirrhosis. Screening based on ultrasound and alpha fetal protein involved a cost of $\$ 219,600$. Screening for all patients with liver cirrhosis is a questionable approach, because it is very expensive and its benefits in terms of patient survival are poor. One way to increase the cost-effectiveness of screening programs according to the authors is to develop more targeted programs with respect to different risk factors.

\section{Discussion}

This study is a review of available evidence about the cost-effectiveness of HCC screening techniques. This review included economic evaluation studies conducted in different contexts. In general, a screening strategy is likely to be cost effective in every setting considered and a semiannual surveillance was shown to be the most cost effective timing strategy. Incidence is the key parameter which determines the cost-effectiveness of HCC screening. ${ }^{1,2,4,9}$ Discrepancies in the results exist when determining the type of technology to be used. US alone or in association with AFP technology is likely to be the most cost effective and the use of CT shows controversial results. Screening should be implemented to detect HCC at an early stage of cirrhosis and it is likely to be not cost effective as the HCC progresses or after liver transplantation. TACE and RA are likely to be not cost effective. Results from Bolondi et $\mathrm{al}^{14,15}$ are likely to be out of the range of cost-effectiveness which, in other studies comparing the same strategies is around $\$ 30,000 / \mathrm{Q} A L Y$. The results are derived from trials and have been conducted by following a societal perspective. However these differences are not crucial to determining the differences in the results which are likely to be determined by the time horizon considered in the ICER evaluation, which is 1 year.

In general, studies were well-written but only partially followed the international guidelines for the conduction of pharmacoeconomic studies. ${ }^{16}$ Probabilistic sensitivity analysis was performed in only a few cases and the presentation of cost-effectiveness acceptability curves (CEAC) was quite rare. Heterogeneity should also be addressed as well as the expected value for perfect information (EVPI) analysis which is crucial to determining the lack of information which should be resolved in further studies. No studies reported considerations about the generalizability of the results in other settings or jurisdictions. There was a lack of randomized controlled trials (RCTs) that could help to address many of the questions about the cost-effectiveness of HCC screening programs in a real setting. In particular, the organization of healthcare is likely to be the key factor which determines the effectiveness and cost-effectiveness of a screening program. Health professionals as well as the network of primary care 
organizations should support screening programs, target the risk populations and implement actions (counseling) to increase the participation of the population in the screening. Those key factors have not been addressed in the existing studies, as the large majority was based on decision models. The need to design specific RCTs to investigate the effectiveness of one single technology or combination of technologies is likely to be clear from this review. RCTs should be designed following a health technology assessment (HTA)-based approach, considering cost-effectiveness as well as organizational, societal, and safety aspects of both the screening techniques and the following treatment.

Clustered randomization is likely to be the most consistent study design as it is likely to capture the health care organization impact on the cost-effectiveness ratio. This review has limitations, some linked to the search being limited to English and Italian, but also setting a time period between January 2000 and March 2011, which may have led to the exclusion of some relevant articles for the purposes of this study. We conclude that screening programs for HCC are cost-effective, allowing an increase in QALY at a sustainable cost. More information is needed about which technology is the most cost effective. The quality of the studies should be improved and the design of new HTA-based clustered RCT is needed for the decision-makers to be better supported.

\section{Disclosure}

The author reports has no conflicts of interest in this work.

\section{References}

1. El-Serag HB, Mason AC. Rising incidence of hepatocellular carcinoma in the United States. N Engl J Med. 1999;340(10):745-750.

2. Chalasani N, Said A, Ness R, Hoen H, Lumeng L. Screening for hepatocellular carcinoma in patients with cirrhosis in the United States: results of a national survey. Am J Gastroenterol. 1999;94(8):2224-2229.
3. Sallie R. Screening for hepatocellular carcinoma in patients with chronic viral hepatitis: can the results justify the effort? Viral Hepatitis Rev. 1995;1:77-95.

4. Deuson RR, Brodovicz KG, Barker L, Zhou F, Euler GL. Economic analysis of a child vaccination project among Asian Americans in Philadelphia, Arch Pediatr Adolesc Med. 2001;155(8):909-914.

5. Yiu-Kuen BD, Ching LL, Man FY. Natural history of hepatitis-related hepatocellular carcinoma. World J Gastroenterol. 2008;14(11): 1652-1656.

6. Heyward WI, Lanier AP, McMaban BK. Screening for primary hepatocellular carcinoma among persons infected with Hepatitis B virus. JAMA. 1985;254(21):3052-3054.

7. Thomas MB, Zhu AX. Hepatocellular carcinoma: the need for progress. J Clin Oncol. 2005;23(13):2892-2899.

8. Bruix J, Sherman M. Management of hepatocellular carcinoma. Hepatology. 2005;42(5):1208-1236.

9. Thompson Coon J, Rogers G, Hewson P, et al. Surveillance of cirrhosis for hepatocellular carcinoma: a cost-utility analysis. Br J Cancer. 2008;98(7):1166-1175. Epub April 1, 2008.

10. Saab S, Ly D, Nieto J, Kanwal F, et al. Hepatocellular carcinoma screening in patients waiting for liver transplantation: a decision analytic model. Liver Transpl. 2003;9(7):672-681.

11. Arguedas MR, Chen VK, Eloubeidi MA, Fallon MB. Screening for hepatocellular carcinoma in patients with hepatitis $C$ cirrhosis: a costutility analysis. Am J Gastroenterol. 2003;98(3):679-690.

12. Naugler WE, Sonnenberg A. Survival and cost-effectiveness analysis of competing strategies in the management of small hepatocellular carcinoma. Liver Transpl. 2010;16(10):1186-1194.

13. Ladabaum U, Cheng SL, Yao FY, Roberts JP. Cost-effectiveness of screening for recurrent hepatocellular carcinoma after liver transplantation. Clinical Transplantation. 2011;25(2):283-291.

14. Bolondi L, Gaiani S, Casali A, Serra C, Piscaglia F. Screening for the early diagnosis of hepatocellular carcinoma: cost-effectiveness analysis. Radiol Med. 1997;94(1-2):4-7.

15. Bolondi L, Sofia S, Siringo S, et al. Surveillance programme of cirrhotic patients for early diagnosis and treatment of hepatocellular carcinoma: a cost-effectiveness analysis. Gut. 2001;48(2):251-259.

16. ISPOR, Country specific pharmacoeconomic guidelines, ISPOR 2006. Available from: http://www.ispor.org/PEguidelines/index.asp. Accessed January 24, 2012.

\section{Publish your work in this journal}

Risk Management and Healthcare Policy is an international, peerreviewed, open access journal focusing on all aspects of public health, policy, and preventative measures to promote good health and improve morbidity and mortality in the population. The journal welcomes submitted papers covering original research, basic science, clinical \& epidemio-

\section{Dovepress}

logical studies, reviews and evaluations, guidelines, expert opinion and commentary, case reports and extended reports. The manuscript management system is completely online and includes a very quick and fair peerreview system, which is all easy to use. Visit http://www.dovepress.com/ testimonials.php to read real quotes from published authors. 